\title{
CÂNCER DE COLO DO ÚTERO: MORTALIDADE EM SANTA CATARINA - BRASIL, 2000 A 2009
}

\author{
Maria Angélica Arzuaga-Salazar1, Maria de Lourdes de Souza², Haimee Emerich Lentz Martins³, Maria \\ Teresa Rogério Locks ${ }^{4}$, Marisa Monticelli ${ }^{5}$, Heloisa Galotti Peixoto ${ }^{6}$
}

\footnotetext{
${ }^{1}$ Doutoranda do Programa de Pós-Graduação em Enfermagem (PEN) da Universidade Federal de Santa Catarina (UFSC). Docente da Facultad de Enfermería da Universidad de Antioquia, Colômbia. E-mail: marzuaga@tone.udea.edu.co

${ }^{2}$ Doutora em Saúde Pública. Docente do PEN/UFSC. Coordenadora Geral da Rede de Promoção do Desenvolvimento da Enfermagem. Santa Catarina, Brasil. E-mail lourdesr@repensul.ufsc.br

${ }^{3}$ Doutoranda do PEN/UFSC. Enfermeira da Secretaria de Estado da Saúde de Santa Catarina (SES/SC). Santa Catarina, Brasil. E-mail haimeelentz@hotmail.com

${ }^{4}$ Mestre em Saúde Pública. Enfermeira diretora de planejamento, avaliação e controle do Sistema Único de Saúde da SES/SC. Santa Catarina, Brasil. E-mail: mlocks@bol.com.br

${ }^{5}$ Doutora em Enfermagem. Docente do Departamento de Enfermagem e do PEN/UFSC. Santa Catarina, Brasil. E-mail: marisa@ nfr.ufsc.br

${ }^{6}$ Mestre em Saúde Pública. Assistente da Gerência de Tecnologia da Informação da SES/SC. Santa Catarina, Brasil. E-mail: helo@saude.sc.gov.br
}

RESUMO: O câncer de colo do útero no Brasil é a terceira neoplasia mais comum em mulheres e apresenta alta mortalidade. O objetivo foi o de avaliar a mortalidade por câncer de colo do útero, ocorrida no Estado de Santa Catarina, no período de 2000 a 2009. Estudo descritivo de base populacional, com a inclusão de todos os óbitos de mulheres, por câncer de colo do útero. Nos dados coletados do Sistema de Informação de Mortalidade, do Ministério da Saúde brasileiro, foram identificadas 1253 mortes de mulheres associadas a esta neoplasia. A taxa de mortalidade calculada variou no período entre 3,6 a 4,9 mortes por 100.000 mulheres, sendo mais elevada em mulheres com sessenta e mais anos. A enfermagem contribui para a construção da cultura de prevenção desta neoplasia, para realização dos testes diagnósticos e para a captação e o seguimento das mulheres de risco pela rede básica de saúde.

DESCRITORES: Neoplasias do colo do útero. Mortalidade. Enfermagem oncológica.

\section{CERVICAL CANCER: MORTALITY IN SANTA CATARINA - BRAZIL, FROM 2000 TO 2009}

\begin{abstract}
Cervical cancer in Brazil is the third most common cancer among women and presents a high mortality rate. The objective of this study was to evaluate cervical cancer mortality occurring in the state of Santa Catarina, from 2000 to 2009. This was a populationbased descriptive study, including all female deaths due to cervical cancer. Data was collected from the Mortality Information System, from the Brazilian Ministry of Health, identifying 1253 female deaths associated with this neoplasm. The mortality rate calculated over the period ranged from 3.6 to 4.9 deaths per 100,000 women, registering more elevated rates among women older than sixty years. Nursing contributed to building a culture of prevention of this neoplasm, to performing diagnostic tests, and to capturing and following-up with at-risk women within primary Brazilian healthcare.
\end{abstract}

DESCRIPTORS: Uterine cervical neoplasms. Mortality. Oncologic nursing.

\section{EL CÁNCER DE CUELLO UTERINO: MORTALIDAD EN SANTA CATARINA - BRASIL, 2000 A 2009}

RESUMEN: El cáncer de cuello uterino en Brasil es la tercera neoplasia más común en mujeres y presenta alta mortalidad. El objetivo del estudio fue evaluar la mortalidad por causa del cáncer de cuello uterino en el Estado de Santa Catarina, en el periodo de 2000 a 2009 . Es un estudio descriptivo, con la inclusión de todas las muertes de mujeres por causa del cáncer de cuello uterino. En los datos recogidos en el Sistema de Información sobre Mortalidad del Ministerio de Salud de Brasil, identificó 1253 muertes de mujeres relacionadas con esta neoplasia. La tasa de mortalidad calculada en el período varió 3,6 a 4,9 muertes por 100.000 mujeres, siendo mayor en las mujeres con más de sesenta años. La enfermería contribuye a construir una cultura de prevención de esta neoplasia, para realización de las pruebas de diagnóstico y para la captura y seguimiento de las mujeres en riesgo por la red atención primaria de salud.

DESCRIPTORES: Neoplasias del cuello uterino. Mortalidad. Enfermería oncológica. 


\section{INTRODUÇÃO}

O Câncer do Colo do Útero (CCU) é uma causa de morte evitável, pois existem tecnologias disponíveis para a sua detecção precoce. Durante a evolução da doença há lesões que podem se constituir em sinais preditivos que representam a probabilidade de cura. ${ }^{1}$

O diagnóstico precoce e o tratamento eficaz da doença são viáveis, pois esta apresenta etapas bem definidas, longo período para a evolução das lesões precursoras, e facilidade de detecção das alterações na fase inicial, o que lhe confere um dos mais altos potenciais de prevenção e cura entre todos os tipos de câncer. ${ }^{2-3}$

Apesar disso, no ano de 2009, o CCU ocupou o terceiro lugar na distribuição proporcional do total de mortes por câncer em mulheres no Brasil, perfazendo $6,4 \%$ de todas as mortes por câncer. ${ }^{4} \mathrm{~A}$ mortalidade por esse câncer no Brasil apresenta-se heterogênea nas diferentes regiões: 8,1 por 100.000 no Norte; 6,7 por 100.000 no Centro-Oeste; 5,8 por 100. 000 no Sul; 4,8 por 100.000 no Nordeste e 4,6 por 100.000 no Sudeste. ${ }^{5}$

A efetividade da detecção precoce do câncer do útero por meio do exame Papanicolaou, associada ao tratamento da lesão intraepitelial, tem resultado em uma redução da incidência do câncer invasor do colo do útero de $90 \%$, produzindo um impacto significativo nas taxas de morbimortalidade. Mas, segundo a Organização Pan-Americana de Saúde (OPAS) e a Organização Mundial de Saúde (OMS), um padrão de qualidade e uma cobertura de rastreamento de 80 a $85 \%$ da população de risco são necessários para se conseguir este efeito. ${ }^{6-7}$

No Brasil, a melhoria da cobertura do exame citológico ainda não foi suficiente para reduzir a mortalidade por câncer do colo do útero em muitas regiões. O diagnóstico tardio ainda é o mais habitual e pode estar relacionado a dificuldades no acesso da população feminina aos serviços e programas de saúde, baixa capacitação dos recursos humanos envolvidos na atenção oncológica, incapacidade do sistema de saúde para absorver a demanda às Unidades de Saúde e dificuldades dos gestores municipais e estaduais em definir e estabelecer um fluxo assistencial com hierarquização dos diversos níveis de atenção. ${ }^{7}$

A qualidade dos dados registrados sobre as causas de morte no Brasil também podem resultar na subenumeração e, em consequência, a obtenção de taxas de mortalidade não representarem corretamente a realidade. Acerca disso, destaca-se que os "dados oficiais sobre mortalidade por câncer do colo do útero no Brasil variam entre 5 e 6 mortes/100.000 mulheres/ano, enquanto que dados da Agência Internacional de Pesquisa do Câncer (International Agency for Research on Cancer - IARC) estimaram que, em 2002, a mortalidade por essa neoplasia no Brasil foi de 10,2 mortes/100.000 mulheres/ano". 8:214

No Estado de Santa Catarina, no ano de 2010, a população era composta por 6.248 .436 pessoas, $\mathrm{e}$ desta, 50,38\% era integrada por mulheres, ou seja, 3.148.076. ${ }^{9}$ Em virtude do contexto apresentado desenvolveu-se um estudo com o objetivo de analisar a mortalidade por câncer de colo do útero ocorrida no Estado de Santa Catarina, no período de 2000 a 2009.

\section{MÉTODO}

Realizou-se estudo descritivo com dados populacionais do Estado de Santa Catarina, localizado na Região Sul do Brasil. Nele, foram incluídas as mortes de mulheres por CCU registradas como ocorridas no Estado de Santa Catarina, em todas as faixas etárias, em cada ano da série histórica de 2000 a 2009. Os dados foram coletados por causa básica e codificados como "C53 Neoplasia maligna do colo do útero", conforme consta na $10^{\text {a }}$ Revisão da Classificação Internacional de Doenças (CID 10). ${ }^{10}$

O estudo foi aprovado pelo Comitê de Ética em Pesquisa em Seres Humanos (CEPSH) da Universidade Federal de Santa Catarina, por meio do parecer consubstanciado - Projeto n ${ }^{\circ}$ 209/2008, muito embora seja realizado com dados secundários, pois os dados acerca das mortes foram coletados no Sistema de Informação de Mortalidade (SIM) do Ministério da Saúde, Brasil, e os relativos à população segundo sexo, faixa etária e macrorregião, no Instituto Brasileiro de Geografia e Estatística (IBGE).4,9

Os dados sobre CCU foram sistematizados por faixa etária, ano a ano, no período de 2000 a 2009, e macrorregião do Estado. Foram calculadas as taxas de mortalidade (número de mulheres que morreram com CCU em relação ao total de mulheres $\times 100.000)$, por faixa etária das mulheres, e macrorregião do Estado, e todos os cálculos foram realizados com o suporte do programa Microsoft Office Excel $2007^{\circ}{ }^{11}$

\section{RESULTADOS}

No Estado de Santa Catarina, Sul do Brasil, no período de 2000 a 2009, foram encontradas no 
Sistema de Informação de Mortalidade 1.253 mortes de mulheres associadas ao CCU. No período, a taxa de mortalidade variou entre 3,6 a 4,9 mortes por 100.000 mulheres.
Nos dados apresentados na tabela 1 se observa, ainda, que a taxa de mortalidade mais baixa foi na faixa etária de zero a 29 anos, e as mais altas, a partir dos cinquenta anos.

Tabela 1 - Taxas de mortalidade por neoplasias de colo do útero (por 100.000 mulheres) por grupo etário. Santa Catarina, Brasil. 2000-2009

\begin{tabular}{ccccccccc}
\hline \multicolumn{7}{c}{ Faixa etária } \\
\cline { 2 - 7 } Ano & $\begin{array}{c}\mathbf{0} \text { a 29 } \\
\text { anos }\end{array}$ & $\begin{array}{c}\mathbf{3 0} \text { a } \mathbf{3 9} \\
\text { anos }\end{array}$ & $\begin{array}{c}\mathbf{4 0} \text { a } \mathbf{4 9} \\
\text { anos }\end{array}$ & $\begin{array}{c}\mathbf{5 0} \text { a } \mathbf{5 9} \\
\text { anos }\end{array}$ & $\begin{array}{c}\mathbf{6 0} \text { a } \mathbf{6 9} \\
\text { anos }\end{array}$ & $\begin{array}{c}\mathbf{7 0} \text { a 79 } \\
\text { anos }\end{array}$ & $\begin{array}{c}\mathbf{8 0} \text { anos e } \\
\text { mais }\end{array}$ & Total \\
\hline $\mathbf{2 0 0 0}$ & 0,4 & 4,3 & 13,1 & 10,6 & 14,3 & 22,8 & 35,0 & 4,9 \\
$\mathbf{2 0 0 1}$ & 0 & 3,8 & 11,4 & 14,7 & 17,8 & 9,2 & 27,5 & 4,6 \\
$\mathbf{2 0 0 2}$ & 0,2 & 5,4 & 9,2 & 11,2 & 11,7 & 14,3 & 3,4 & 4,0 \\
$\mathbf{2 0 0 3}$ & 0,6 & 2,8 & 9,7 & 12,4 & 15,1 & 12,8 & 20,1 & 4,1 \\
$\mathbf{2 0 0 4}$ & 0,6 & 4,0 & 9,2 & 11,8 & 11,4 & 12,7 & 19,8 & 4,0 \\
$\mathbf{2 0 0 5}$ & 1,0 & 3,3 & 6,8 & 14,5 & 18,7 & 23,4 & 19,2 & 4,4 \\
$\mathbf{2 0 0 6}$ & 1,0 & 3,8 & 8,6 & 8,7 & 8,2 & 13,3 & 28,4 & 3,6 \\
$\mathbf{2 0 0 7}$ & 0,2 & 4,1 & 8,5 & 12,1 & 16,1 & 18,0 & 15,8 & 4,8 \\
$\mathbf{2 0 0 8}$ & 0,7 & 4,1 & 7,3 & 8,1 & 14,9 & 18,4 & 8,5 & 4,3 \\
$\mathbf{2 0 0 9}$ & 0,2 & 2,8 & 9,4 & 10,6 & 10,5 & 14,9 & 32,3 & 4,6 \\
\hline
\end{tabular}

Fonte: Sistema de Informações sobre Mortalidade e IBGE. ${ }^{4,9}$

A taxa de mortalidade, quando calculada por macrorregiões do Estado de Santa Catarina, apresentou variabilidade no período, no interior da região e em relação às demais regiões. A va- riação apresentada nas taxas não demonstrou uma tendência ou regularidade de ocorrência da mortalidade (Tabela 2).

Tabela 2 - Taxas de mortalidade por neoplasias de colo do útero (por 100.000 mulheres) por macrorregiões do Estado de Santa Catarina, Brasil. 2000-2009

Macrorregiões de Santa Catarina

\begin{tabular}{|c|c|c|c|c|c|c|c|c|c|c|}
\hline Ano & 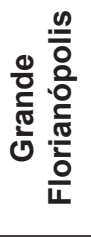 & $\overline{\bar{\varpi}}$ & 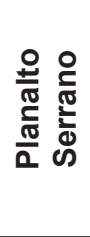 & 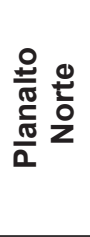 & 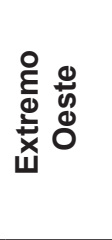 & 을 $\frac{0}{d}$ & $\begin{array}{l}\frac{0}{0} \cdot \frac{\pi}{\pi} \\
\frac{0}{\pi}\end{array}$ & 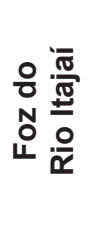 & 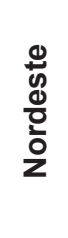 & 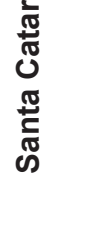 \\
\hline 2000 & 6,7 & 3,0 & 5,5 & 3,0 & 3,9 & 5,0 & 4,6 & 8,0 & 5,2 & 4,9 \\
\hline 2001 & 6,8 & 4,4 & 5,5 & 1,2 & 5,4 & 4,2 & 3,7 & 5,8 & 3,1 & 4,6 \\
\hline 2002 & 2,7 & 3,8 & 3,4 & 2,4 & 6,2 & 3,1 & 5,1 & 6,6 & 2,7 & 4,0 \\
\hline 2003 & 5,1 & 0,7 & 4,1 & 2,3 & 5,6 & 3,5 & 4,4 & 3,7 & 6,2 & 4,1 \\
\hline 2004 & 4,0 & 2,1 & 3,4 & 7,0 & 4,4 & 2,1 & 5,2 & 4,4 & 4,5 & 4,0 \\
\hline 2005 & 5,5 & 3,0 & 7,3 & 1,1 & 3,2 & 3,0 & 4,3 & 7,9 & 5,3 & 4,4 \\
\hline 2006 & 3,5 & 1,6 & 8,0 & 0,0 & 1,2 & 3,0 & 5,8 & 5,7 & 5,2 & 3,6 \\
\hline 2007 & 4,3 & 4,2 & 2,0 & 5,0 & 3,8 & 3,6 & 5,9 & 5,9 & 7,0 & 4,8 \\
\hline 2008 & 5,1 & 2,7 & 4,6 & 5,0 & 4,7 & 1,0 & 5,1 & 3,9 & 5,8 & 4,3 \\
\hline 2009 & 2,9 & 3,8 & 3,3 & 3,3 & 5,5 & 4,7 & 6,0 & 3,4 & 7,4 & 4,6 \\
\hline
\end{tabular}




\section{DISCUSSÃO}

As taxas de mortalidade calculadas para o Estado de Santa Catarina, no período de 2000 a 2009, não tiveram nenhum processo de correção e, por isto mesmo, podem estar subestimadas. Os resultados de pesquisa com dados secundários, na realidade brasileira, incorrem no risco da subestimação, tendo em vista a qualidade do registro dos dados e, por conseguinte, as taxas calculadas podem divergir de outras fontes. ${ }^{5,8}$

A mortalidade observada no Estado de Santa Catarina, no período estudado, apresentou uma variação que demonstra uma tendência de pouca flutuação. Dados similares são registrados para outras regiões do Brasil. ${ }^{12}$

As taxas observadas no Estado de Santa Catarina diferem das encontradas para as capitais dos Estados da Região Nordeste do Brasil, no período de 1996 a 2005, porém se aproximam dos achados para o interior daqueles Estados. ${ }^{5}$ Também diferem do que foi observado na Região Norte, no período de 2002-2004. ${ }^{13}$ Há diferenças regionais importantes e estas estão associadas com vários fatores. ${ }^{14}$

As diferenças ocorrem no contexto interno da região, ao longo do tempo, e, também, em relação a outros caracteres como as áreas urbanas e rurais, capital e interior, e outros conglomerados definidos pelos pesquisadores. No presente estudo, cabe destacar que a Região Nordeste de Santa Catarina foi a que apresentou taxas mais elevadas em relação à da Grande Florianópolis.

As taxas de mortalidade por CCU aumentam progressivamente com a idade das mulheres, e isto está registrado por outros pesquisadores no Brasil e no mundo. ${ }^{1,15-17}$

A mortalidade observada em todas as faixas etárias demonstra que a prevenção, primária e secundária, deve ser reforçada. A prevenção secundária inclui o conjunto de ações que promovem o diagnóstico precoce da doença e o seu tratamento imediato, aumentando a possibilidade de cura, melhorando a qualidade de vida e a sobrevida e diminuindo a mortalidade por câncer. ${ }^{18}$ Assim, quando os profissionais de saúde realizam suas atividades em relação à detecção precoce do câncer, têm como objetivo prevenir o câncer de colo do útero invasor, detectando e tratando mulheres com lesões intraepiteliais de alto grau. ${ }^{19}$

A efetividade da detecção precoce pode ser avaliada pela redução da incidência do CCU, bem como da mortalidade das mulheres. No Brasil, há resultados como os observados no Estado do Para- ná, de redução da mortalidade por câncer do colo do útero, a partir do rastreamento e do aumento na cobertura do exame Papanicolaou. ${ }^{20}$

As provas de detecção precoce fazem parte dos programas de detecção e controle dessa doença, entre as quais a mais utilizada é a prova PAP, conhecida também como teste de Papanicolaou, Pap Smear, exame de citologia, citologia cérvicouterina, citologia cervical, citologia vaginal, ou simplesmente, "preventivo". Esta técnica é de baixo custo e sua finalidade é avaliar as células de colo do útero e detectar modificações celulares, mediante análise citológica microscópica das células escamadas de colo do útero.

Cabe destacar que estão sendo utilizadas e testadas as provas de ADN de HPV, de detecção visual, a olho nu ou com colposcópio, das mudanças de colo do útero, após a utilização de Ácido Acético (IVA) e Solução Iodada de Lugol (IVSL), e técnicas fotográficas de colo do útero. Esta última é conhecida também como cervicografia digital de colo do útero. ${ }^{21} \mathrm{Em}$ muitos países, a citologia ou as provas de ADN de HPV, ou ambas, são usadas para a detecção sistemática de CCU e para acompanhamento após o diagnóstico. Nos países de baixa renda e sem boa infraestrutura sanitária, a inspeção visual de colo do útero com ácido acético ou solução iodada de Lugol resulta na detecção de possíveis lesões, tratáveis imediatamente com crioterapia - estratégia conhecida como "ver e tratar". ${ }^{22}$ Diversos estudos vêm sendo desenvolvidos com a finalidade de testar a sensibilidade e a especificidade das provas disponíveis, como os estudos coordenados pela IARC, na Índia, no Peru, na África do Sul e na Tailândia. ${ }^{19,23}$ Os objetivos estão centrados no desenvolvimento de estratégias e provas custoefetivas e facilmente acessíveis para mulheres de poucos recursos econômicos. ${ }^{24}$

\section{CONCLUSÕES}

As taxas de mortalidade por câncer de colo do útero em Santa Catarina apresentam variação entre as regiões estudadas e no interior de cada região, entre os distintos anos.

As taxas de mortalidade aumentam nas idades mais avançadas e isto demonstra a necessidade de assistência preventiva, ao longo da vida da mulher.

A enfermagem contribui para reduzir a mortalidade por câncer do colo do útero quando promove, na Rede Básica de Saúde, a construção da cultura de prevenção, a ampliação do acesso 
das mulheres aos serviços de saúde, a realização dos testes diagnósticos, e a captação e seguimento adequado das mulheres de risco.

\section{REFERÊNCIAS}

1. Mendonça VG, Lorenzato FRB, Mendonça JG, Menezes TC, Guimarães MJB. Mortalidade por câncer do colo do útero: características sociodemográficas das mulheres residentes na cidade de Recife, Pernambuco. Rev Bras Ginecol Obstet [online]. 2008 Mai. [acesso 2010 Out 29]; 30(5). Disponível em: http:/ / www.scielo.br/scielo.php?script $=$ sci arttext\&pid=S0100-72032008000500007\&lng=en\& nrm=iso

2. Derossi SA, Paim JS, Aquino E, Silva LMV. Evolução da mortalidade e anos potenciais de vida perdidos por câncer cérvico-uterino em Salvador (BA), 19791997. Rev Bras Cancerol. 2001; 47(2):163-70.

3. Fonseca LAM, Ramacciotti AS, Eluf Neto J. Tendência da mortalidade por câncer do útero no Município de São Paulo entre 1980 e 1999. Cad Saúde Pública. 2004; 20(1):136-42.

4. Ministério da Saúde (BR). Departamento de Informática do Sistema Único de Saúde (DATASUS). Sistema de informação de mortalidade [página da Internet]. 2009 [acesso 2009 Dez 15]. Disponível em: tabnet.datasus.gov.br/cgi/idb2006/matriz.htm

5. Gamarra CJ, Valente JG, Azevedo e Silva G. Correção da magnitude da mortalidade por câncer do colo do útero no Brasil, 1996-2005. Rev Saude Publica. 2010; 44(4):629-38.

6. Ministério da Saúde (BR). Instituto Nacional de Câncer. Periodicidade de realização do exame preventivo do câncer do colo do útero: normas e recomendações do INCA. Rev Bras Cancerol. 2002; 48(1):13-5.

7. Ministério da Saúde (BR). Instituto Nacional de Câncer. Nomenclatura brasileira para laudos cervicais e condutas preconizadas - recomendações para profissionais de saúde. 2a ed. Rio de Janeiro: INCA; 2006.

8. Zeferino LC. O desafio de reduzir a mortalidade por câncer do colo do útero Rev Bras Ginecol Obstet. 2008; 30(5):213-5.

9. Instituto Brasileiro de Geografia e Estatística (IBGE). Censo Demográfico 2010. Rio de Janeiro: IBGE; 2010 [acesso 2011 Abr]. Disponível em: http:// www.censo2010.ibge.gov.br/sinopse/index. php?uf $=42 \&$ dados $=0$.

10. Organização Mundial da Saúde (OMS). Classificação Estatística Internacional de Doenças e Problemas Relacionados à Saúde - 10 ${ }^{\mathrm{a}}$ Revisão. Centro Colaborador da Organização Mundial da Saúde para a Classificação de Doenças em Português. São Paulo: OMS; 1995.
11. Excel, MO. Parte do produto Microsoft Office Professional Edição 2007 ed: Microsoft Corporation; Copyright $^{(\mathrm{C})}$ 1985-2007. SP1 ed; 2007.

12. Alves CMM, Guerra MR, Bastos RR. Tendência de mortalidade por câncer de colo de útero para o Estado de Minas Gerais, Brasil, 1980-2005. Cad Saúde Pública [online]. 2009 Ago [acesso 2010 Out 29]; 25(8):1693-700. Disponível em: http://www.scielosp.org/scielo.php?script=sci arttext\&pid=S0102-311X2009000800005\&lng=en

13. Boing AF, Vargas SAL, Boing AC. A carga das neoplasias no Brasil: mortalidade e morbidade hospitalar entre 2002-2004. Rev Assoc Med Bras 2007;53(4):317-22.

14. Thuler LC. Mortalidade por câncer do colo do útero no Brasil. Rev Bras Ginecol Obstet. 2008;30(5):216-8 2

15. Organización Mundial de la Salud (OMS). Mejor detección, tratamiento y vacunas asequibles para prevenir muertes por cancer cervicouterino. Temas de salud 2009 [Internet] [acesso 2009 Out 21]. Disponível em: http://new.paho.org/hq/index. php?option $=$ com_content\&task $=$ view\&id $=142 \& I t$ emid=259\&lang $=$ es

16. Donoso SE, Cuello FM, Villarroel L. Reducción de la mortalidad por cáncer cérvico uterino en Chile, 1990-2003. Rev Chil Obstet ginecol [online]. 2006 [acesso 2010 Out 29]; 71(5):307-12. Disponível em: http:/ / www.scielo.cl/scielo.php?script=sci arttext\&pid=S0717-75262006000500003\&lng=es

17. Sepúlveda VP, González CF, Napolitano RC, Roncone DE, Cavada CG. Cáncer de cuello uterino: sobrevida a 3 y 5 años en hospital San José. Rev Chil Obstet Ginecol [online]. 2008 [acesso 2010 Out 29]; 73(3):151-4. Disponível em: http://www. scielo.cl $/$ scielo.php? script $=$ sci_arttext\&pid $=$ S071775262008000300003\&lng=es

18. Angus J, Paszat L, McKeever P, Trebilcock A, Shivji F, Edwards B. Trajetórias para o diagnóstico e o tratamento do cancêr de mama: explorando as relações sociais na demora do diagnóstico. Texto Contexto Enferm [online]. 2007 Out-Dez [acesso 2009 Set 20]; 16(4):591-8. Disponível em: http:// www.scielo.br/pdf/tce/v16n4/a02v16n4.pdf

19. Sankaranarayanan R, Somanathan T, Santos C, Muwonge R, Ngoma T. Odesafio decontrolar ocâncer do colo do útero no mundo em desenvolvimento. In: Coelho FRG, Soares FA, Foch J, Fregnani JH, Zeferino LC, Villa LL, Federido MH, Novaes PE, Costa RL. Câncer do colo do útero. São Paulo: Tecmedd; 2008. p. 104-13.

20. Guerra MR, Gallo CVM, Azevedo GSM. Risco de câncer no Brasil: tendências e estudos epidemiológicos mais recentes. Rev Bras Cancerol. 2005; 51(3):227-34.

21. Franco ES, Hyppólito SB, Franco R, Oriá M, Almeida PC, Pagliuca LM, et al. Critérios de positividade para cervicografia digital: melhorando a sensibilidade do 
diagnóstico do câncer cervical. Cad. Saúde Pública [online]. 2008 Nov [acesso 2009 Set 20]; 24(11):265360. Disponível em: http://www.scielo.br/scielo. php?script=sci_arttext\&pid=S0102-311X200800110 0020\&lng=en\&nrm=iso

22. Ministério da Saúde (BR). Instituto Nacional de Câncer. Estimativa 2010: incidência de câncer no Brasil [Internet]. Rio de Janeiro; 2009 [acesso 2010 Fev 10]. Disponível em: http:/ / www1.estado.com. $\mathrm{br} / \mathrm{redac} /$ manual.html

23. Sankaranarayanan R, Esmy PO, Muwonge R, Swaminathan R, Shanthakumari S, Fayette J-M,
Cherian J. Effect of visual screening on cervical cancer incidence and mortality in Tamil Nadu, India: a cluster-randomised trial. Lancet [onlinr]. 2007 Ago. [acesso 2009 Set 10]; 370(9585):398-406. Disponível em: http://www.thelancet.com/journals/lancet/ article/PIIS0140-6736(07)61195-7/fulltext

24. Alliance for cervical cancer prevention. 10 hallazgos y recomendaciones esenciales para programas eficaces de Tamizaje y tratamiento del cáncer cervicouterino. In: Prevención del cáncer cervicouterino: ficha descriptiva [Internet]. 2007 [acesso 2010 Jan 14]. Disponível em: http:/ / www.alliance-cxca.org/ espanol/ACCP_recs_2007_factsheet_Spanish.pdf 